\title{
Exploring the risk-factor association between depression and incident stroke: a systematic review and meta-analysis
}

This article was published in the following Dove Press journal:

Neuropsychiatric Disease and Treatment

18 December 2014

Number of times this article has been viewed

\author{
Kristian Barlinn' \\ Jessica Kepplinger' \\ Volker Puetz' \\ Ben M Illigens ${ }^{2}$ \\ Ulf Bodechtel' \\ Timo Siepmann'
}

'Department of Neurology, University Hospital Carl Gustav Carus Dresden, University of Technology Dresden, Dresden, Germany; ${ }^{2}$ Department of Neurology, Beth Israel Deaconess Medical Center, Harvard Medical School, Boston, MA, USA

Correspondence: Kristian Barlinn Department of Neurology, University Hospital Carl Gustav Carus, 74 Fetscherstrasse, Dresden 01307, Germany

Tel +4935 I 4583565

Fax +49 35I 4584365

Email kristian.barlinn@uniklinikumdresden.de

\begin{abstract}
There is growing evidence that depression increases the risk of incident stroke. However, few studies have considered possible residual confounding effects by preexistent cerebrovascular and cardiac diseases. Therefore, we synthesized data from cohort studies to explore whether depressed individuals free of cerebrovascular and cardiac diseases are at higher risk of incident stroke. We searched the electronic databases PubMed and Medline for eligible cohort studies that examined the prospective association between depression and first-ever stroke. A random-effects model was used for quantitative data synthesis. Sensitivity analyses comprised cohort studies that considered a lag period with exclusion of incident strokes in the first years of follow-up to minimize residual confounding by preexistent silent strokes and excluded cardiac disease at baseline. Overall, we identified 28 cohort studies with 681,139 participants and 13,436 (1.97\%) incident stroke cases. The pooled risk estimate revealed an increased risk of incident stroke for depression (relative risk 1.40, 95\% confidence interval [CI] $1.27-1.53 ; P<0.0001$ ). When we excluded incident strokes that occurred in the first years of follow-up, the prospective association between depression and incident stroke remained significant (relative risk 1.64, 95\% CI 1.27-2.11; $P<0.0001)$. This positive association also remained after we considered only studies with individuals with cardiac disease at baseline excluded (relative risk $1.43,95 \% \mathrm{CI}$ $1.19-1.72 ; P<0.0001)$. The prospective association of depression and increased risk of firstever stroke demonstrated in this meta-analysis appears to be driven neither by preexistence of clinically apparent cerebrovascular and cardiovascular diseases nor by silent stroke.
\end{abstract}

Keywords: stroke, prestroke, depression, risk factor

\section{Introduction}

While for many years, supported by the results of two recent high-quality meta-analyses, it has been assumed that depression constitutes a possible risk factor for stroke, the previous hypothetic concept of "vascular depression" is fueling a new debate in the scientific community. ${ }^{1-3}$ The theory is that the presence of subclinical cerebrovascular diseases may induce neurobiological changes in brain regions, including the dorsal cortex and limbic structures, and therewith predispose, precipitate, or perpetuate depressive syndromes and at the same time occurrence of stroke. ${ }^{3}$ The findings of a recent large-scale cohort study including more than 10,000 people further substantiate this hypothesis, when no association between depression and incident stroke was evident after considering only cerebrovascular events that occurred beyond the first years of follow-up. ${ }^{4}$ The latter approach may ensure that results are not biased by the fact that people who have had a clinically silent or apparent stroke immediately prior to study initiation have a higher risk of developing depression in the short term (ie, reverse causality). ${ }^{5}$ 
To date, a large portion of all conducted cohort studies have not focused on stroke-free participants as the target population. For instance, the meta-analysis by Pan et al identified seven of 28 included studies that enrolled people with a history of stroke, increasing the chance that the findings were biased by reverse causality. ${ }^{2}$ Less attention has been given to other vascular comorbidities like cardiac disease in this matter. Apart from the probable causal relationship with cardiac disease causing depression and vice versa, it is a well-known fact that patients with cardiac disease have more than twice the odds of having a stroke than those without cardiac diasese. ${ }^{6-8}$ Moreover, these patients generally have a higher burden of vascular risk factors potentiating the manifestation of subclinical cerebrovascular disease that in turn may increase the possibility of residual confounding in longitudinal cohort studies. ${ }^{9}$

Since recently published reviews and meta-analyses, results of other longitudinal cohort studies investigating the prospective association between depression and incident stroke have been published. Modified inclusion criteria derived from conclusions and recommendations of previous research have been implemented to minimize study heterogeneity and biases, and now allow more in-depth conclusions on the potential risk-factor association between depression and stroke. Therefore, we aimed to update and enlarge the earlier knowledge base on the risk of a first-ever stroke in people with depression compared to people without depression by performing a systematic review and meta-analysis of cohort studies. We particularly explored the question of whether people with depression and free of both cerebrovascular and cardiac diseases are at higher risk of incident stroke or whether such association might rather result from reverse causality.

\section{Materials and methods}

\section{Literature search and study selection}

We performed a systematic literature search using the PubMed and Medline electronic databases. The text words "depression" and "stroke" with the use of the Boolean operator "AND" were used to identify relevant studies that examined the prospective association between depression and incident stroke. We exclusively chose these two text words and their corresponding MeSH (Medical Subject Heading) terms ("depressive disorder" OR "depressive" AND "disorder" OR "depressive disorder" OR “depression" OR “depression" AND "stroke") to ensure coverage of all potentially eligible articles stored in the searched electronic databases. In addition, we manually searched the bibliographies of recently published opinion statements, reviews, and meta-analyses that addressed the risk-factor association between depression and stroke to identify further articles of interest..$^{1,2,10,11} \mathrm{We}$ only considered articles published in the English language for further review and excluded duplicates.

The title and abstract of the identified articles were screened according to the eligibility criteria detailed in the next section by an independent reviewer (KB). Full texts were retrieved and reviewed for definite eligibility. Any ambiguities concerning the eligibility of a full text were discussed with a second independent investigator (JK) to reach a final consensus. In the case of missing information or any obscurities, the lead authors of the identified articles were contacted for clarification. The literature search covered studies from the earliest date available until our last search on August 24, 2014. Our systematic review and meta-analysis complied with the Preferred Reporting Items for Systematic Reviews and Meta-Analyses (PRISMA) guidelines. ${ }^{12}$

\section{Eligibility criteria}

According to our formulated PICOS (participants, interventions, comparators, outcomes, and study design) question, ${ }^{12}$ an original epidemiological study was included if 1) participants were nonhospitalized adults with no history of stroke or transient ischemic attack (TIA) at the time of study initiation, 2) the exposure (intervention) variable was defined as depression, whereas assessment of depression had to be prospectively performed at baseline and based upon an objective measure like a neuropsychological mood scale or neuropsychiatric evaluation that complied with the Diagnostic Statistical Manual for Mental Disorders (DSM)-III/IV or the International Classification of Diseases (ICD)-7-10, 3) the comparison group consisted of participants without depression at the time of study initiation, 4) the dichotomous outcome event of interest was a first-ever stroke during the follow-up period, including fatal and nonfatal ischemic stroke, TIA, and intracerebral hemorrhage (hereinafter referred as "all stroke"), and 5) the study design was a community-, population-, or registry-based longitudinal cohort study reporting relative effect estimates, such as hazard ratios (HRs), relative risk (RRs), and odds ratios (ORs).

\section{Data items}

The following data items were extracted from the included original full-text articles and entered into a predetermined Excel (Microsoft, Redmond, WA, USA) spreadsheet: 1) article information (name of first author, publication year, journal), 2) study design (population-level, study geographical location, length of follow-up, methods used for assessment of the exposure [eg, applied mood scale, neuropsychiatric 
face-to-face evaluation] and outcome measures [eg, review of hospital discharge records, death certificates], cohort size, variables included in the final adjusted model), 3) results (population characteristics including age and sex, number of incident cases registered during follow-up in the exposed and unexposed participants, adjusted effect estimates and respective $95 \%$ confidence intervals [CIs] arising from the most fully adjusted models, and risk estimates by strata of interest [eg, cardiac disease, age-group, lag period]).

\section{Assessment of risk of bias in individual studies}

Each individual cohort study was reviewed for study quality and potential sources of bias by two independent investigators (KB and JK) using the Newcastle-Ottawa Scale for observational studies recommended by the Cochrane Collaboration. ${ }^{13,14}$ Briefly, each included study was rated according to three grouping items: selection of cohort, comparability of cohort, and assessment of outcome. High-quality choices were scored with a star, with a maximum of nine stars achievable for all eight subitems (one star for each numbered item within the selection and outcome categories; a maximum of two stars for comparability). Of note, adjustment for the most relevant stroke risk factors (eg, arterial hypertension and age), minimum length of follow-up of 10 years, dropout rate less than $20 \%$, and exposure, as well as outcome ascertained other than self-report (eg, linked primary record sources), were considered high-quality choices.

\section{Synthesis of results and additional analyses}

Statistical analyses were performed with the use of the software package Stata (Version 12.1; StataCorp, College Station, TX, USA) and the user-written package Metan for meta-analyses. For simplicity reasons, we reported RR with 95\% CI as the common effect estimate for the association between the exposure and the incident cases (hereinafter referred to as overall RR). However, to minimize statistical heterogeneity, the primary and some of the additional analyses were carried out separately for studies reporting $\mathrm{HR}$ and $\mathrm{OR} / \mathrm{RR}$. The $\mathrm{OR}$ and $\mathrm{RR}$ reflect the risk at a given time point, and both can be considered interchangeable when the absolute number of cases is small $(<10 \%) .{ }^{15}$ Since the event rates were below $10 \%$ in the included studies, we merged OR and RR for this purpose. The most fully adjusted effect estimates from the included studies were combined using the DerSimonian and Laird random-effects model. ${ }^{16}$ Assuming substantial heterogeneity among a set of largescale cohort studies gathered from the published literature, this method was chosen a priori, as it cannot be anticipated that variation in observed effect estimates is exclusively a result of sampling error (that would be the assumption for a fixed-effect model). ${ }^{17}$

A test of between-study statistical heterogeneity was performed using the Cochran's $Q$ statistic (with significant heterogeneity set at $P<0.1$ ), and the percentage of variation across studies attributable to heterogeneity was described with the $I^{2}$ statistic. ${ }^{18}$ An $I^{2}$ value of 0 indicates no, 25\%-50\% low, $50 \%-75 \%$ moderate, and $>75 \%$ high observed heterogeneity. We ascertained potential sources of between-study heterogeneity and the robustness of the obtained results by several sensitivity and subgroup analyses. Statistical significance was set at $P<0.05$. If an included study reported stratified effect estimates, these were pooled using the inverse-variance fixed-effect or DerSimonian and Laird random-effects model (whichever applicable). We assessed publication bias by visual inspection of the funnel plot and the use of Egger's linear regression and Begg's rank correlation tests. ${ }^{19,20}$ The Duval and Tweedie trim-and-fill method was applied to identify and adjust for significant small-study effects. ${ }^{21}$

\section{Results \\ Systematic review}

As detailed in the flowchart in Figure 1, a total of 6,090 titles/ abstracts were retrieved from the electronic literature and 62 from the bibliographies of published literature. Of these, we independently reviewed 61 full-text articles for eligibility and retained 28 articles for our quantitative data synthesis., ${ }^{4,-48}$

Since the previously published meta-analyses covering a literature search until May 2011, ${ }^{1,2}$ nine more published studies investigating the prospective relationship between depression and stroke with 412,699 participants and 6,559 incident stroke cases were identified according to our eligibility criteria and included in our quantitative data synthesis. 4,25,26,29,30,34,36,41,42 All included studies were designed as prospective community- or population-based cohort studies, except two that comprised registry-based cohorts. ${ }^{29,42}$ The total number of participants in the included studies was 681,139 with 13,436 (1.97\%) incident stroke cases observed during a total follow-up ranging from 3 to 29 years. One study did not report the exact number of incident cases. ${ }^{46}$ Diagnosis of depression was largely based on a self-rating depression scale $(n=24)$, with the Center for Epidemiological Studies - Depression (CES-D) being the most commonly used assessment scale $(n=11)$. In only four studies, the baseline depression diagnosis originated from a direct evaluation through a psychologist, psychiatrist, or other physician according to ICD or DSM criteria. ${ }^{29,34,35,42}$ 


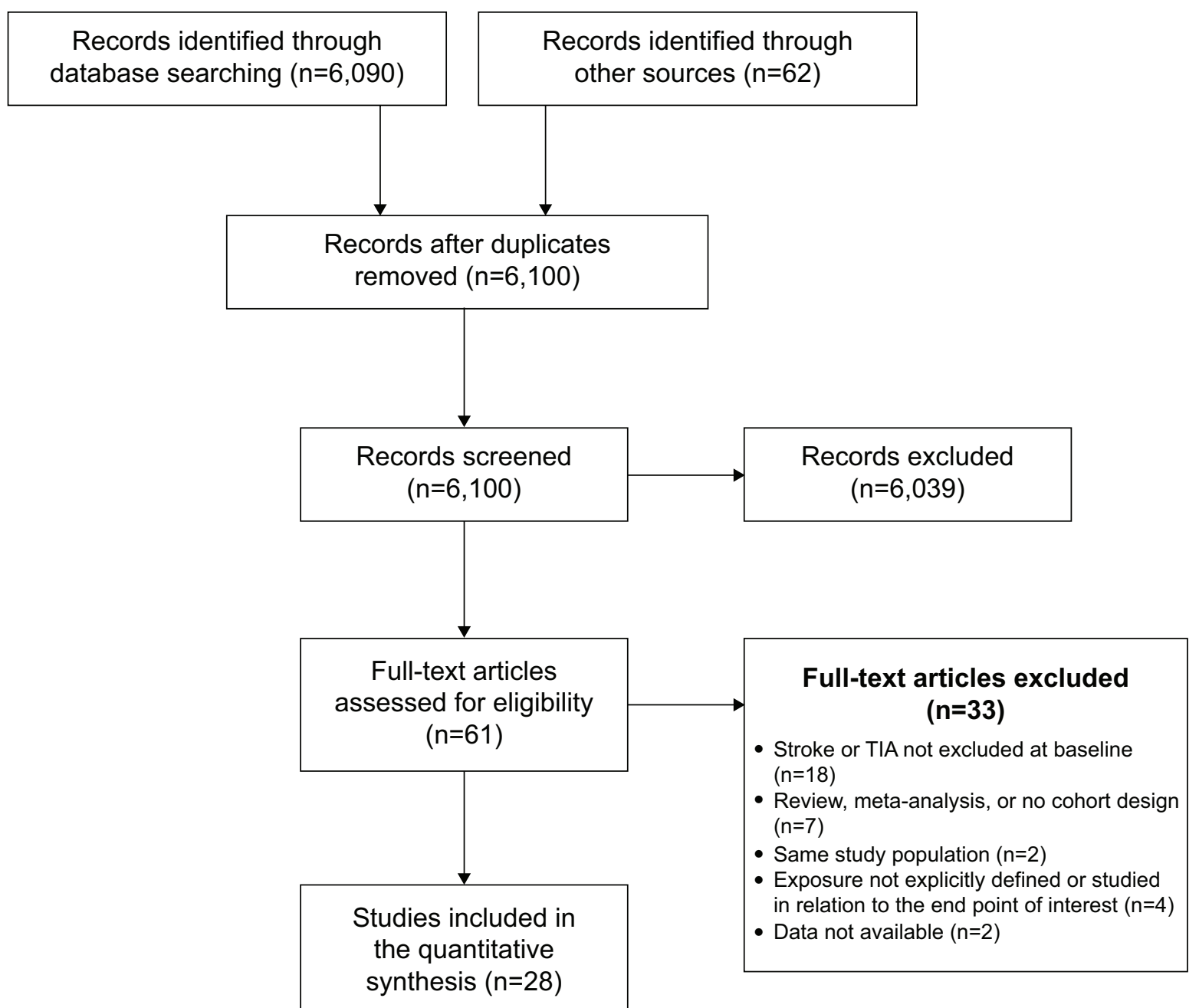

Figure I Flow diagram of study selection for the quantitative data synthesis. Abbreviation: TIA, transient ischemic attack.

Most of the studies used medical records and death certificates, to some extent in combination with self- or proxy reporting, for the diagnosis of stroke. In only four studies, the diagnosis of (nonfatal) stroke solely relied on self- or proxy reporting. ${ }^{23,27,30,33}$ Most studies restricted the end point to ischemic and hemorrhagic stroke ( $\mathrm{n}=15)$, ${ }^{4,23,24,26,28-31,36,37,40,41,44,47,48}$ two studies to ischemic stroke and TIA, ${ }^{22,42}$ and eleven studies captured all stroke (ischemic, hemorrhagic, and TIA) end points. . $^{25,27,32-35,38,39,43,45,46}$ With regard to stroke survival, three studies recorded only fatal stroke. ${ }^{24,28,32}$ Fifteen studies excluded participants with cardiac disease at baseline a priori or in additional analyses, ${ }^{25,26,28,31,32,34,36-38,41,42,44,46-48}$ of which six had not been included in previous meta-analyses. In terms of effect estimates, six studies reported RRs, ${ }^{31,33,35,38,39,47}$ 19 HRs, ${ }^{4,22-28,32,36,37,40-46,48}$ and three ORs. ${ }^{29,30,34}$ A full summary of the included studies, with an assessment of study quality by the Newcastle-Ottawa Scale, is provided in Table 1.
To minimize the possibility of reverse causality, seven studies considered a lag period by excluding the first years of follow-up (first 2 years, $n=2$; first 3 years, $n=2$; first 5 years, $n=3), 4,24,31,32,36,37,41$ and one study considered only participants free of cerebrovascular disease who had received a depression diagnosis at least 10 years before baseline. ${ }^{42}$ Six studies were primarily conducted in participants aged $\geq 65$ years, ${ }^{22,23,32,35,39,41}$ and seven studies in participants $<65$ years. ${ }^{4,26,28,30,36,37,44}$ The age of participants in the remainder of studies overlapped these age strata; however, two studies provided additional analyses for effect estimates stratified by age ( $<65$ and $\geq 65$ years). ${ }^{27,43}$ Three studies exclusively reported effect estimates stratified by race/sex (white men, white women, black persons) and age ( $<65$ and $\geq 65$ as well as $65-74$ and $\geq 75$ years, respectively) strata. ${ }^{23,31,43}$ From these studies, pooled estimates were used for the current analysis. An additional analysis defining 


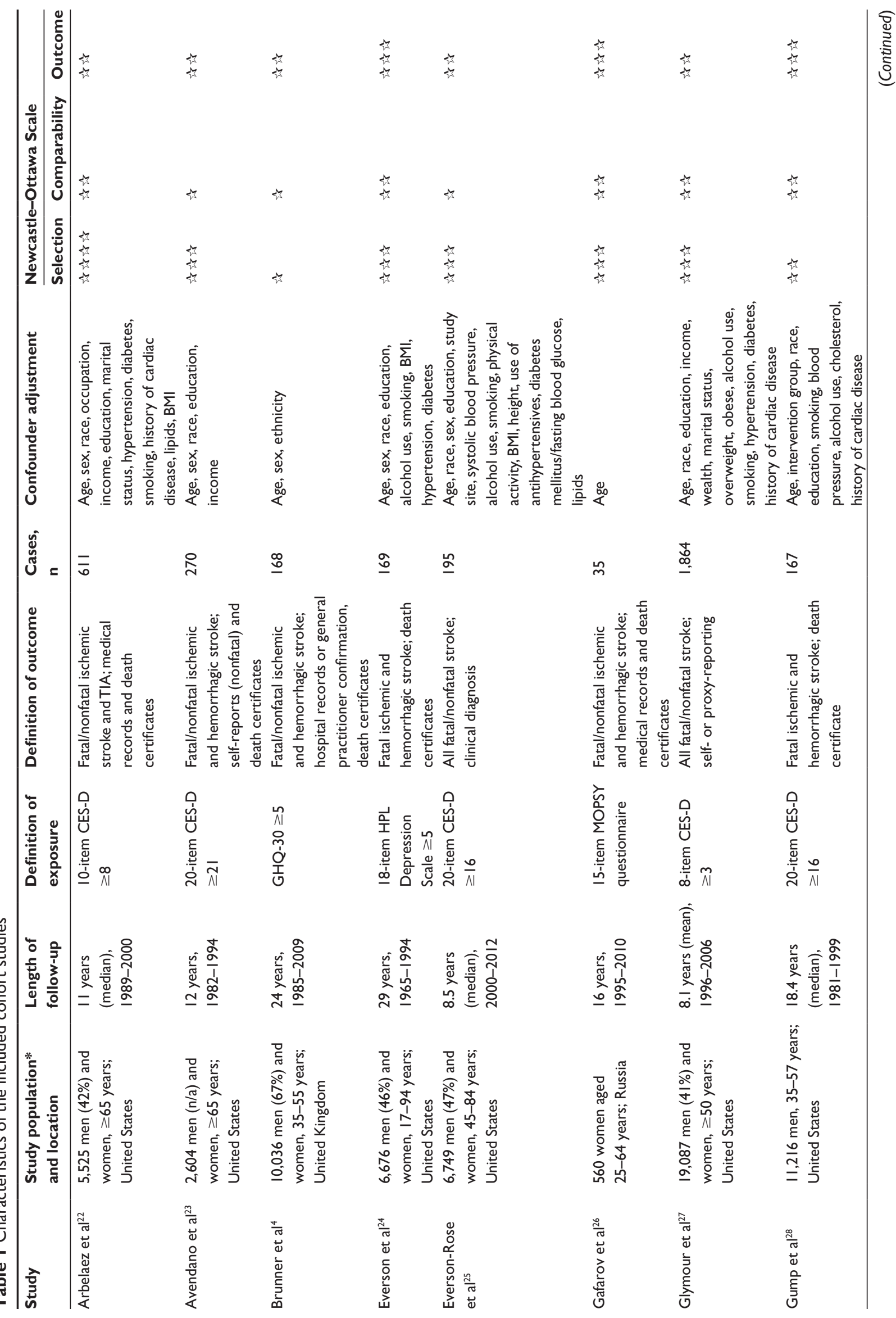









\begin{tabular}{|c|c|c|c|c|}
\hline 资 & 资 & 资 & 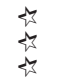 & 如 \\
\hline 资 & 望 & 资 & 资 & 资 \\
\hline z & 资 & 资 & 资 & 资 \\
\hline
\end{tabular}

กำ 은

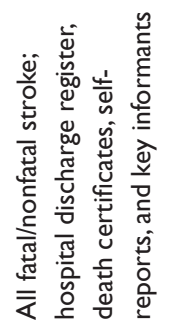

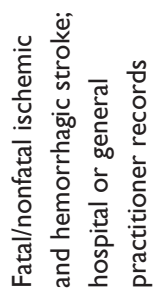

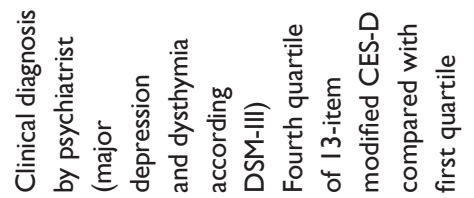

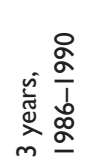

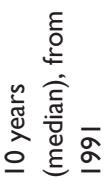

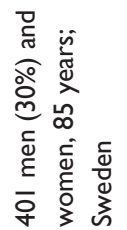

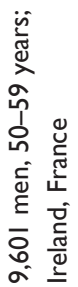

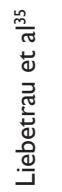

ฐิ

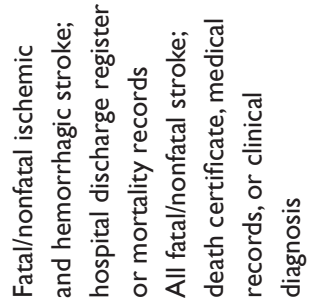

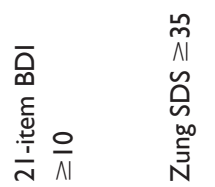

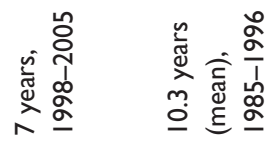

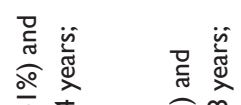

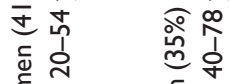

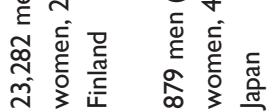

o용

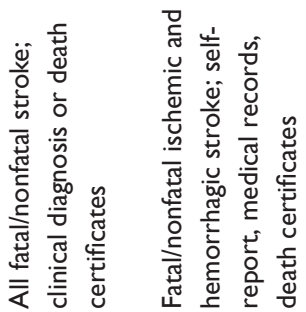

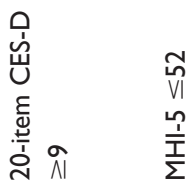

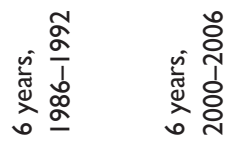

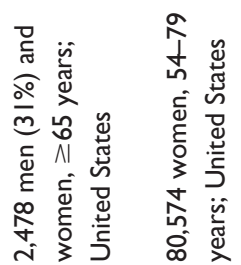

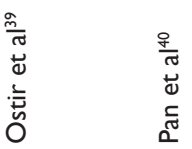




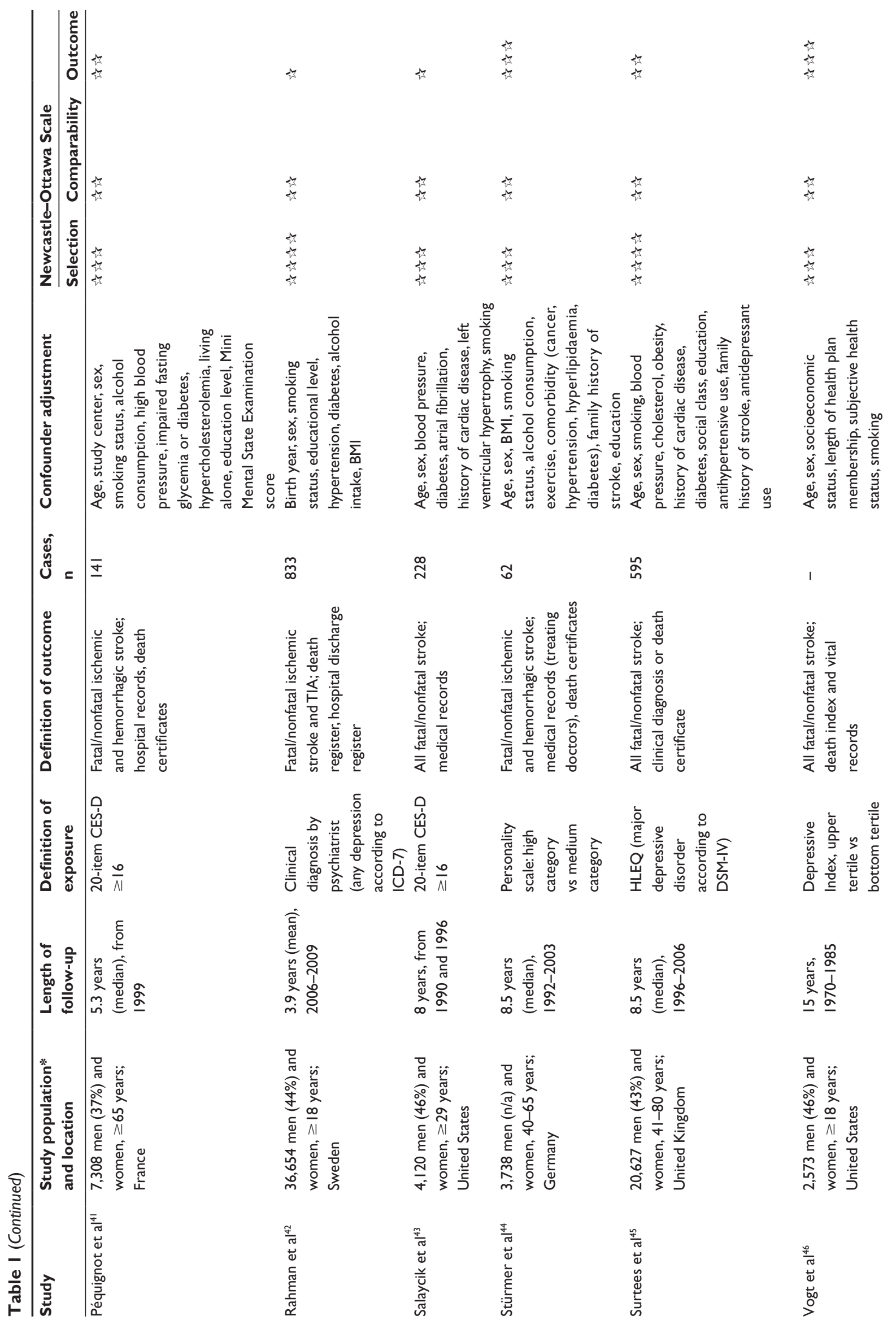




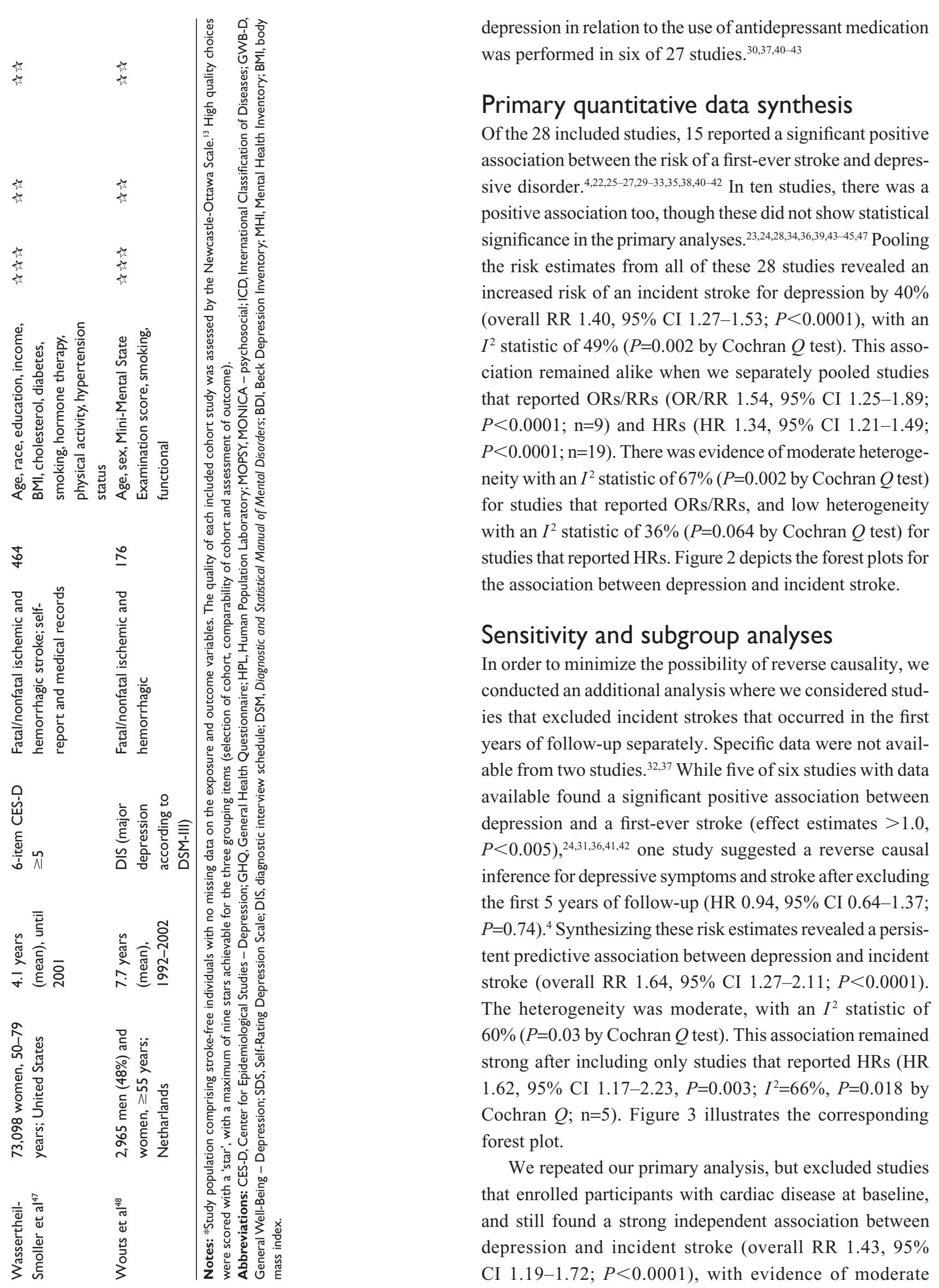




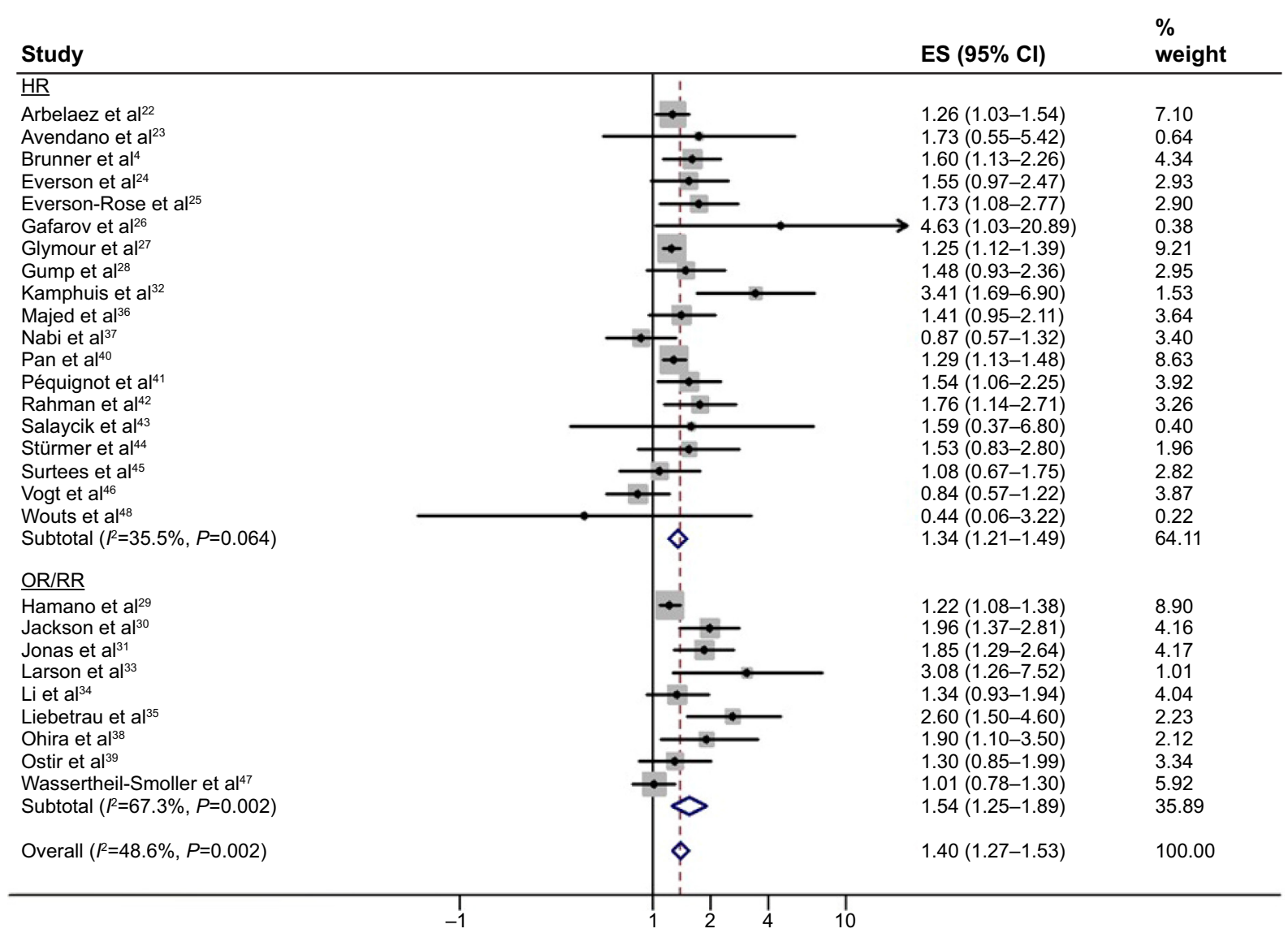

Figure 2 Forest plot showing the pooled adjusted risk estimates for first-ever stroke in depressed individuals.

Note: Weights are from random effects analysis.

Abbreviations: $\mathrm{ES}$, effect size; $\mathrm{Cl}$, confidence interval; $\mathrm{HR}$, hazard ratio; OR, odds ratio; $\mathrm{RR}$, relative risk.

heterogeneity ( $I^{2}=58 \%, P=0.003$ by Cochran $Q$ test). Analyzing studies by preferred reported risk estimates did not change the results. The pooled OR/RR was $1.42(95 \%$ CI 1.03-1.96, $P<0.034 ; \mathrm{n}=4)$, and the pooled HR was 1.45 (95\% CI 1.13-1.85, $P<0.003 ; \mathrm{n}=11$ ), both with evidence of moderate heterogeneity $\left(I^{2}=67 \%, P=0.027\right.$ by Cochran $Q$, and $I^{2}=58 \%, P=0.008$ by Cochran $Q$, respectively). The exclusion of studies that focused on either fatal or nonfatal strokes did not alter the results either (overall RR 1.36, 95\% CI 1.24-1.50, $P<0.0001 ; I^{2}=46 \%, P=0.007$ by Cochran $Q$ ). A strong prospective association between depression and incidence of a first-ever stroke remained after focusing on studies that assessed all stroke subtypes (ie, ischemic and hemorrhagic stroke, TIA) as the outcome measure (overall RR $1.50,95 \%$ CI $1.21-1.86, P<0.0001 ; I^{2}=62 \%, P=0.004$ by Cochran $Q$ ). To minimize misclassification bias, we removed studies that primarily defined the exposure variable by self-reporting, and found a slightly higher risk of incident stroke for clinically diagnosed depression at baseline (overall RR $1.64,95 \%$ CI $1.21-2.24, P=0.002 ; I^{2}=68 \%, P=0.015$ by Cochran $Q$ ). Similar results to those obtained by the primary analysis were found when restricting the data synthesis to a minimum cohort size of 5,000 participants (overall RR $1.33,95 \%$ CI $1.23-1.44, P<0.0001 ; I^{2}=35 \%, P=0.076$ by Cochran $Q$ ) and a minimum duration of follow-up of 5 years (overall RR 1.41, 95\% CI 1.27-1.56, $P<0.0001 ; I^{2}=42 \%$, $P=0.018$ by Cochran $Q$ ). Excluding the two registry-based cohort studies from the primary analysis did not change the results (overall RR 1.40, 95\% CI 1.26-1.55, $P<0.001$; $I^{2}=48 \%, P=0.004$ by Cochran $Q$ ).

When we stratified the included studies by age, it appeared that the $\geq 65$-year (overall RR $1.45,95 \%$ CI $1.20-1.75$, $P<0.0001 ; I^{2}=55 \%, P=0.029$ by Cochran $Q$ ) age-group had a slightly lower risk than the $<65$-year (overall RR $1.59,95 \%$ CI $1.35-2.09, P<0.0001 ; I^{2}=52 \%, P=0.035$ by Cochran $Q$ ) agegroup. Extending the definition of depression to the additional or exclusive use of antidepressants resulted in an elevation of the prospective risk of stroke (overall RR 1.60, 95\% CI $1.23-2.09, P<0.0001 ; I^{2}=64 \%, P=0.026$ by Cochran $Q$ ).

\section{Publication bias}

The funnel plot appeared slightly asymmetric, with evidence of small-study effects (ie, tendency that smaller studies show larger treatment effects) demonstrated by both 


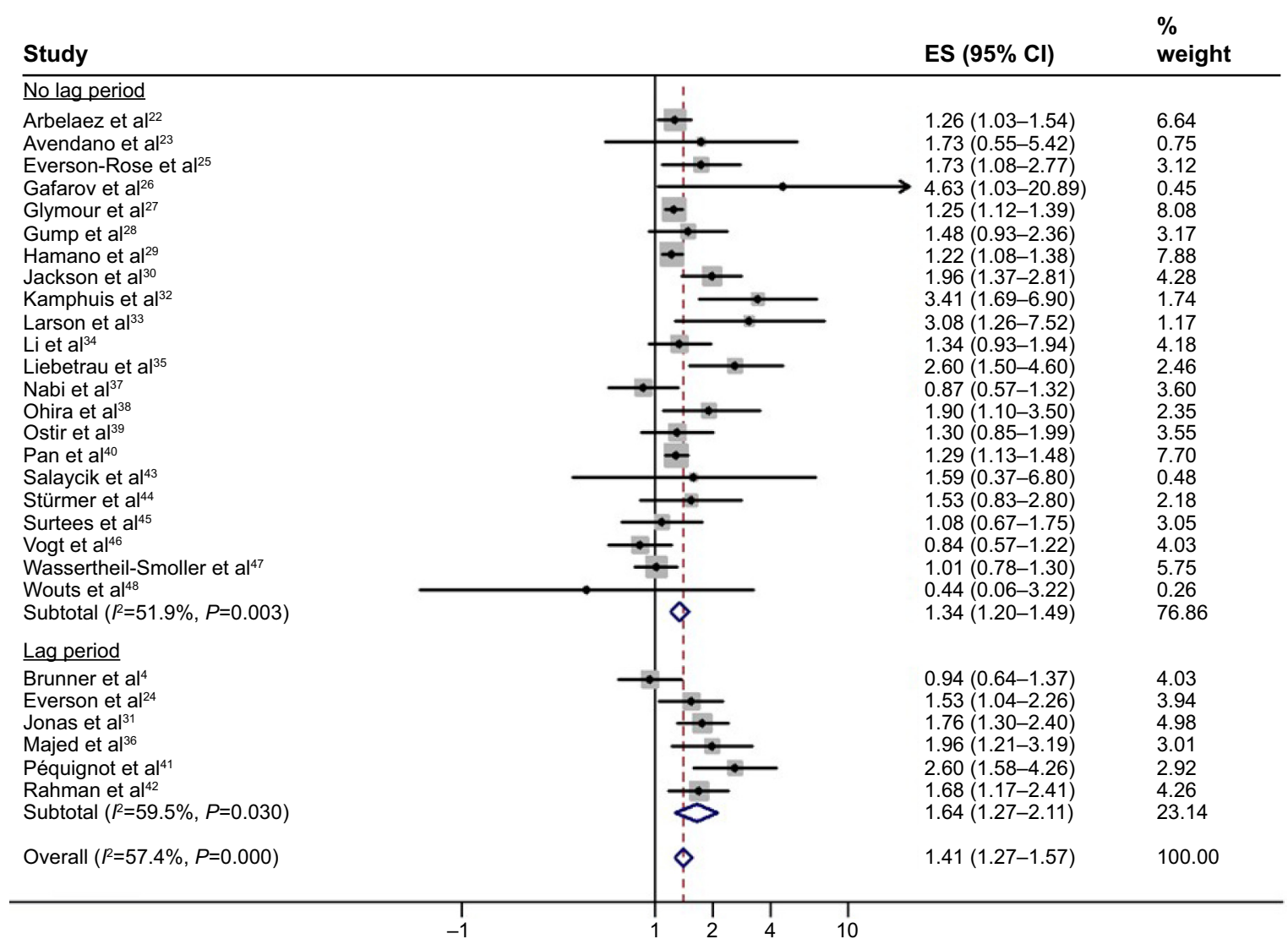

Figure 3 Forest plot showing the pooled adjusted risk estimates for first-ever stroke in depressed individuals according to the analytic approach used in the included study. Lag period indicates that stroke cases that occurred in the first years of follow-up were excluded from the analysis to minimize the possibility of reverse causality.

Note: Weights are from random effects analysis.

Abbreviations: $\mathrm{ES}$, effect size; $\mathrm{Cl}$, confidence interval.

Begg's $(z=1.78, P=0.075)$ and Egger's $(P=0.013)$ tests. We conducted a sensitivity analysis where we imputed eight theoretically missing studies in our meta-analysis using Duval and Tweedie's trim-and-fill method. The recalculation of the pooled effect estimate revealed a slightly attenuated but still significantly increased risk of incident stroke for depression (overall RR 1.26, 95\% CI 1.14-1.40; $P<0.0001$ ). There was no evidence of significant publication bias for studies that reported HR (Begg's $P=0.35$, Egger's $P=0.17$ ) and substantial evidence of bias for studies that reported OR/ RR ( $P=0.026$ by Egger's test). However, the latter studies still yielded a significant association after adjusting for smallstudy effects (OR/RR 1.27, 95\% CI 1.03-1.56; $P=0.026)$.

\section{Discussion}

The major finding of this meta-analysis comprising almost 700,000 participants, consistent with previous analyses, is that depression increases the risk of first-ever stroke by $40 \%$ in the general population. Moreover, our results relating depression to incident stroke are corroborated by recently published similar pooled estimates with an increased overall risk of stroke (34\% and $45 \%$, respectively), yet we included nine new studies, adding more than 400,000 participants. ${ }^{1,2}$ This prospective association appears to be driven neither by the preexistence of clinically apparent cerebro- and cardiovascular disease nor by silent stroke.

While our synthesized results suggest a possible causal association between depression and stroke, recently analyzed data from a large prospective cohort study contradict this contention by showing a nullified incident risk of stroke once a different analytic approach was applied. ${ }^{4}$ In this study, the risk of incident stroke was significantly elevated by $60 \%$ for depressed individuals, yet no such association was evident with exclusion of incident events that occurred in the first 5 years of follow-up. This analytic "lag period" approach was chosen to avoid the impact of possible reverse causality between preexisting silent cerebrovascular disease and depression, based on the vascular depression concept. ${ }^{49,50}$ However, this cohort study was limited by the absence of adjustment for vascular risk factors, use of a nonspecific depression screening scale, and an upper age limit of study participants of 55 years. ${ }^{4}$ In contrast, several previous cohort studies did not show significant influence of a lag period on the prospective association between the risk of incident stroke 
and depression. ${ }^{24,31,36,41,42}$ This is also reflected by the results of our synthesized subanalysis on studies that accounted for residual confounding by excluding the initial follow-up period showing a nearly identical overall (41\%) and even pronounced risk once a lag period was defined, as opposed to studies with no lag period (64\% and 34\%, respectively). Also, the fact that most studies we included in our meta-analysis adjusted properly for well-established vascular risk factors further substantiates the notion that the association between depression and stroke could be independent of coexisting vascular diseases.

In concordance with the original vascular depression concept, which specifically proposed an association between cerebral small-vessel disease and depression in the elderly, a recent meta-analysis confirmed cerebral small-vessel disease being fourfold more common in patients with late- than early onset depression. ${ }^{3,51}$ Another more recent meta-analysis on this subject reported similar though weaker results. ${ }^{52}$ However, the development of cerebral small-vessel disease increases with age and is related to an increased risk of stroke. ${ }^{9}$ Therefore, viewed in conjunction with the vascular depression concept, it could be anticipated that particularly the elderly suffering from depression have an increased risk of stroke. Still, we found a balanced risk of stroke across groups stratified by age (even slightly lower in absolute numbers for the $\geq 65$-year than the $<65$-year age-group), and one study included in our meta-analysis even suggested a higher risk of stroke in younger people with depression. ${ }^{43}$ Specifically, data originating from the Framingham Heart Study showed that depressive symptoms were significantly associated with a fourfold-increased risk of a first-ever ischemic stroke in individuals younger than 65 years but not in those older than or equal to 65 years. This discrepancy of balanced (or even increased) risk of stroke in younger depressed individuals and the increasing risk of stroke with age in the general population may be partially explained by the fact that depressive individuals less commonly adhere to medication than nondepressed people, and consequently primary stroke prevention may be compromised in depressed individuals. ${ }^{53,54}$ Moreover, unhealthy lifestyle, including smoking, unfavorable food, and lack of physical activity, is also associated with depression and in turn an increased risk of stroke..$^{55-59}$

Consistent with our finding of a balanced risk of stroke between young and elderly individuals with depression, a previous systematic review of 23 etiological, phenomenological, and outcome studies did not find any identifiable distinguishing clinical features between early onset and late-onset depression. ${ }^{60}$ Additionally, it might be speculated that an increased stroke risk in depressed people may arise from pharmacological interferences with platelet aggregation, as recently discussed for antidepressant medication and even suggested by the increased stroke risk (60\%) yielded from our subanalysis of six studies that defined depression in relation to the use of antidepressant medication. ${ }^{61}$ On the other hand, a recent study did not confirm an increased risk of stroke in individuals with manic or bipolar disorder, a mental illness also known to be associated with unhealthy lifestyle behavior and a pronounced burden of vascular risk factors. ${ }^{62-64}$ This dissonant increase of stroke risk in depressed but not manic or bipolar individuals might suggest that the underlying cerebral pathomechanisms might be specific for depression. Based on the findings of previous studies, these mechanisms might include cerebral inflammation, dysregulation of the hypothalamic-pituitary-adrenal axis, increased platelet reactivity, and autonomic dysfunction. ${ }^{65}$

Our quantitative data synthesis is subject to limitations, mostly associated with clinical and methodological diversity among the included studies, as shown by the moderate heterogeneity in our pooled data set. Clinical heterogeneity is given by the variety of self-reporting mood scales used in the studies included in our analysis, potentially introducing misclassification and information bias. This may particularly apply to participants prescribed antidepressant medication possibly obscuring the actual prevalence of depression at baseline. Another shortcoming relates to the fact that most of the published studies defined the outcome measures as any stroke subtype instead of focusing on a specific subtype (eg, ischemic or hemorrhagic stroke) or etiology. Therefore, stroke subtype- or etiology-specific differences in the relationship between depression and stroke might remain undetected in our synthesized analysis. Particularly given that in young adults, causes of stroke are less frequently attributed to cerebrovascular diseases compared with the elderly, but comprise a broad spectrum of nonatherosclerotic causes (eg, arterial dissection, hypercoagulable state, nonatherosclerotic arteriopathies, or cryptogenic), our finding of balanced stroke risk in different age-groups must be considered with caution. ${ }^{66}$ This also relates to hemorrhagic stroke, which has a variety of underlying age-dependent etiologies itself.

Even though we excluded studies that had enrolled participants with a known history of stroke in order to minimize confounding effects arising from potential preexisting cerebrovascular disease, it remains speculative to what extent unknown subclinical cerebrovascular disease might have influenced stroke risk in our analysis. Many studies, however, have consistently shown that particularly severe strokes with 
prominent functional disability predict the development of depression. ${ }^{5,67}$ Conversely, it might be speculated that stroke survivors clinically less severely affected may have a lower, if any, risk of developing a depression. Nevertheless, we reduced the probability of coexisting undetected cerebrovascular disease by performing a sensitivity analysis, removing studies that included patients with a history of cardiac disease, and yielded similar results.

\section{Conclusion}

Whether the consistent association of depression and increased risk of first-ever stroke demonstrated in this metaanalysis is indicative of depression-induced neurovascular pathology or is rather attributed to an interplay between two mutually enhancing conditions needs to be further elucidated in long-term studies. These studies should focus on specific stroke subtypes and preferably enroll young individuals free of cerebrovascular and cardiovascular diseases and provide longitudinal cerebral structural and functional assessment.

\section{Disclosure}

The authors report no conflicts of interest in this work.

\section{References}

1. Dong JY, Zhang YH, Tong J, Qin LQ. Depression and risk of stroke: a meta-analysis of prospective studies. Stroke. 2012;43(1):32-37.

2. Pan A, Sun Q, Okereke OI, Rexrode KM, Hu FB. Depression and risk of stroke morbidity and mortality: a meta-analysis and systematic review. JAMA. 2011;306(11):1241-1249.

3. Alexopoulos GS, Meyers BS, Young RC, Campbell S, Silbersweig D, Charlson M. 'Vascular depression' hypothesis. Arch Gen Psychiatry. 1997;54(10):915-922.

4. Brunner EJ, Shipley MJ, Britton AR, et al. Depressive disorder, coronary heart disease, and stroke: dose-response and reverse causation effects in the Whitehall II cohort study. Eur J Prev Cardiol. 2014;21(3): 340-346.

5. Hackett ML, Pickles K. Part I: Frequency of depression after stroke: an updated systematic review and meta-analysis of observational studies. Int J Stroke. Epub 2014 Aug 12.

6. Hare DL, Toukhsati SR, Johansson P, Jaarsma T. Depression and cardiovascular disease: a clinical review. Eur Heart J. 2014;35(21): 1365-1372.

7. Lichtman JH, Froelicher ES, Blumenthal JA, et al. Depression as a risk factor for poor prognosis among patients with acute coronary syndrome: systematic review and recommendations: a scientific statement from the American Heart Association. Circulation. 2014;129(12):1350-1369.

8. O’Donnell MJ, Xavier D, Liu L, et al. Risk factors for ischaemic and intracerebral haemorrhagic stroke in 22 countries (the INTERSTROKE study): a case-control study. Lancet. 2010;376(9735):112-123.

9. Pantoni L. Cerebral small vessel disease: from pathogenesis and clinical characteristics to therapeutic challenges. Lancet Neurol. 2010;9(7):689-701.

10. Scott KM. Depression, anxiety and incident cardiometabolic diseases. Curr Opin Psychiatry. 2014;27(4):289-293.

11. Ramasubbu R, Patten SB. Effect of depression on stroke morbidity and mortality. Can J Psychiatry. 2003;48(4):250-257.
12. Moher D, Liberati A, Tetzlaff J, Altman DG. Preferred reporting items for systematic reviews and meta-analyses: the PRISMA statement. PLoS Med. 2009;6(7):e1000097.

13. Wells GA, Shea B, O'Connell D, et al. The Newcastle-Ottawa Scale (NOS) for assessing the quality if nonrandomized studies in meta-analyses. 2005. Available from: http://www.ohri.ca/programs/ clinical_epidemiology/oxford.htm. Accessed August 30, 2014.

14. Higgins JP, Green S, editors. Cochrane Handbook for Systematic Reviews of Interventions. Version 5.1.0. Oxford: National Institute for Health Research; 2011.

15. Davies HT, Crombie IK, Tavakoli M. When can odds ratios mislead? BMJ. 1998;316(7136):989-991.

16. DerSimonian R, Laird N. Meta-analysis in clinical trials. Control Clin Trials. 1986;7(3):177-188.

17. Borenstein M, Higgins JP. Meta-analysis and subgroups. Prev Sci. 2013; 14(2):134-143.

18. Higgins JP, Thompson SG, Deeks JJ, Altman DG. Measuring inconsistency in meta-analyses. BMJ. 2003;327(7414):557-560.

19. Harbord RM, Egger M, Sterne JA. A modified test for small-study effects in meta-analyses of controlled trials with binary endpoints. Statistics in Medicine. 2006;25(20):3443-3457.

20. Begg CB, Mazumdar M. Operating characteristics of a rank correlation test for publication bias. Biometrics. 1994;50(4):1088-1101.

21. Duval S, Tweedie R. Trim and fill: a simple funnel-plot-based method of testing and adjusting for publication bias in meta-analysis. Biometrics. 2000;56(2):455-463.

22. Arbelaez JJ, Ariyo AA, Crum RM, Fried LP, Ford DE. Depressive symptoms, inflammation, and ischemic stroke in older adults: a prospective analysis in the cardiovascular health study. J Am Geriatr Soc. 2007;55(11):1825-1830.

23. Avendano M, Kawachi I, Van Lenthe F, et al. Socioeconomic status and stroke incidence in the US elderly: the role of risk factors in the EPESE study. Stroke. 2006;37(6):1368-1373.

24. Everson SA, Roberts RE, Goldberg DE, Kaplan GA. Depressive symptoms and increased risk of stroke mortality over a 29-year period. Arch Intern Med. 1998;158(10):1133-1138.

25. Everson-Rose SA, Roetker NS, Lutsey PL, et al. Chronic stress, depressive symptoms, anger, hostility, and risk of stroke and transient ischemic attack in the multi-ethnic study of atherosclerosis. Stroke. 2014;45(8):2318-2323.

26. Gafarov VV, Panov DO, Gromova EA, Gagulin IV, Gafarova AV. The influence of depression on risk development of acute cardiovascular diseases in the female population aged 25-64 in Russia. Int J Circumpolar Health. 2013;72:21223.

27. Glymour MM, Maselko J, Gilman SE, Patton KK, Avendaño M. Depressive symptoms predict incident stroke independently of memory impairments. Neurology. 2010;75(23):2063-2070.

28. Gump BB, Matthews KA, Eberly LE, Chang YF. Depressive symptoms and mortality in men: results from the Multiple Risk Factor Intervention Trial. Stroke. 2005;36(1):98-102.

29. Hamano T, Li X, Lönn SL, et al. Depression, stroke and gender: evidence of a stronger association in men. J Neurol Neurosurg Psychiatry. Epub 2014 Jun 26.

30. Jackson CA, Mishra GD. Depression and risk of stroke in midaged women: a prospective longitudinal study. Stroke. 2013;44(6):1555-1560.

31. Jonas BS, Mussolino ME. Symptoms of depression as a prospective risk factor for stroke. Psychosom Med. 2000;62(4):463-471.

32. Kamphuis MH, Kalmijn S, Tijhuis MA, et al. Depressive symptoms as risk factor of cardiovascular mortality in older European men: the Finland, Italy and Netherlands Elderly (FINE) study. Eur J Cardiovasc Prev Rehabil. 2006;13(2):199-206.

33. Larson SL, Owens PL, Ford D, Eaton W. Depressive disorder, dysthymia, and risk of stroke: thirteen-year follow-up from the Baltimore Epidemiologic Catchment Area Study. Stroke. 2001;32(9):1979-1983.

34. Li CT, Bai YM, Tu PC, et al. Major depressive disorder and stroke risks: a 9-year follow-up population-based, matched cohort study. PLoS One. 2012;7(10):e46818. 
35. Liebetrau M, Steen B, Skoog I. Depression as a risk factor for the incidence of first-ever stroke in 85-year-olds. Stroke. 2008;39(7): 1960-1965.

36. Majed B, Arveiler D, Bingham A, et al. Depressive symptoms, a timedependent risk factor for coronary heart disease and stroke in middleaged men: the PRIME study. Stroke. 2012;43(7):1761-1767.

37. Nabi H, Kivimäki M, Suominen S, Koskenvuo M, Singh-Manoux A, Vahtera J. Does depression predict coronary heart disease and cerebrovascular disease equally well? The Health and Social Support Prospective Cohort Study. Int J Epidemiol. 2010;39(4):1016-1024.

38. Ohira T, Iso H, Satoh S, et al. Prospective study of depressive symptoms and risk of stroke among Japanese. Stroke. 2001;32(4):903-908.

39. Ostir GV, Markides KS, Peek MK, Goodwin JS. The association between emotional well-being and the incidence of stroke in older adults. Psychosom Med. 2001;63(2):210-215.

40. Pan A, Okereke OI, Sun Q, et al. Depression and incident stroke in women. Stroke. 2011;42(10):2770-2775.

41. Péquignot R, Tzourio C, Péres K, et al. Depressive symptoms, antidepressants and disability and future coronary heart disease and stroke events in older adults: the Three City Study. Eur J Epidemiol. 2013; 28(3):249-256.

42. Rahman I, Humphreys K, Bennet AM, Ingelsson E, Pedersen NL, Magnusson PK. Clinical depression, antidepressant use and risk of future cardiovascular disease. Eur J Epidemiol. 2013;28(7):589-595.

43. Salaycik KJ, Kelly-Hayes M, Beiser A, et al. Depressive symptoms and risk of stroke: the Framingham Study. Stroke. 2007;38(1):16-21.

44. Stürmer T, Hasselbach P, Amelang M. Personality, lifestyle, and risk of cardiovascular disease and cancer: follow-up of population based cohort. BMJ. 2006;332(7554):1359.

45. Surtees PG, Wainwright NW, Luben RN, Wareham NJ, Bingham SA, Khaw KT. Psychological distress, major depressive disorder, and risk of stroke. Neurology. 2008;70(10):788-794.

46. Vogt T, Pope C, Mullooly J, Hollis J. Mental health status as a predictor of morbidity and mortality: a 15-year follow-up of members of a health maintenance organization. Am J Public Health. 1994;84(2):227-231.

47. Wassertheil-Smoller S, Shumaker S, Ockene J, et al. Depression and cardiovascular sequelae in postmenopausal women. The Women's Health Initiative (WHI). Arch Intern Med. 2004;164(3):289-298.

48. Wouts L, Oude Voshaar RC, Bremmer MA, Buitelaar JK, Penninx BW, Beekman AT. Cardiac disease, depressive symptoms, and incident stroke in an elderly population. Arch Gen Psychiatry. 2008;65(5):596-602.

49. Marquis GS, Habicht JP, Lanata CF, Black RE, Rasmussen KM. Association of breastfeeding and stunting in Peruvian toddlers: an example of reverse causality. Int J Epidemiol. 1997;26(2):349-356.

50. Taylor WD, Aizenstein HJ, Alexopoulos GS. The vascular depression hypothesis: mechanisms linking vascular disease with depression. Mol Psychiatry. 2013;18(9):963-974.

51. Herrmann LL, Le Masurier M, Ebmeier KP. White matter hyperintensities in late life depression: a systematic review. J Neurol Neurosurg Psychiatry. 2008;79(6):619-624.
52. Wang L, Leonards CO, Sterzer P, Ebinger M. White matter lesions and depression: a systematic review and meta-analysis. $J$ Psychiatr Res. 2014;56:56-64.

53. Fiedorowicz JG. Depression and cardiovascular disease: an update on how course of illness may influence risk. Curr Psychiatry Rep. 2014;16(10):492.

54. Maidment R, Livingston G, Katona C. Just keep taking the tablets: adherence to antidepressant treatment in older people in primary care. Int J Geriatr Psychiatry. 2002;17(8):752-757.

55. Compton MT, Daumit GL, Druss BG. Cigarette smoking and overweight/obesity among individuals with serious mental illnesses: a preventive perspective. Harv Rev Psychiatry. 2006;14(4):212-222.

56. Savolainen J, Kautiainen H, Miettola J, Niskanen L, Mäntyselkä P. Low quality of life and depressive symptoms are connected with an unhealthy lifestyle. Scand J Public Health. 2014;42(2):163-170.

57. Roshanaei-Moghaddam B, Katon WJ, Russo J. The longitudinal effects of depression on physical activity. Gen Hosp Psychiatry. 2009; 31(4):306-315.

58. Kilian R, Becker T, Krüger K, Schmid S, Frasch K. Health behavior in psychiatric in-patients compared with a German general population sample. Acta Psychiatr Scand. 2006;114(4):242-248.

59. Tikk K, Sookthai D, Monni S, et al. Primary preventive potential for stroke by avoidance of major lifestyle risk factors: the European Prospective Investigation into Cancer and Nutrition-Heidelberg cohort. Stroke. 2014;45(7):2041-2046.

60. Grayson L, Thomas A. A systematic review comparing clinical features in early age at onset and late age at onset late-life depression. $J$ Affect Disord. 2013;150(2):161-170.

61. Chen Y, Guo JJ, Li H, Wulsin L, Patel NC. Risk of cerebrovascular events associated with antidepressant use in patients with depression: a population-based, nested case-control study. Ann Pharmacother. 2008; 42(2):177-184.

62. Nilsson FM, Kessing LV. Increased risk of developing stroke for patients with major affective disorder - a registry study. Eur Arch Psychiatry Clin Neurosci. 2004;254(6):387-391.

63. de Almeida KM, Moreira CL, Lafer B. Metabolic syndrome and bipolar disorder: what should psychiatrists know? CNS Neurosci Ther. 2012;18(2):160-166.

64. Cerimele JM, Katon WJ. Associations between health risk behaviors and symptoms of schizophrenia and bipolar disorder: a systematic review. Gen Hosp Psychiatry. 2013;35(1):16-22.

65. Penninx BW, Milaneschi Y, Lamers F, Vogelzangs N. Understanding the somatic consequences of depression: biological mechanisms and the role of depression symptom profile. BMC Med. 2013;11:129.

66. Ferro JM, Massaro AR, Mas JL. Aetiological diagnosis of ischaemic stroke in young adults. Lancet Neurol. 2010;9(11):1085-1096.

67. Ayerbe L, Ayis S, Wolfe CD, Rudd AG. Natural history, predictors and outcomes of depression after stroke: systematic review and metaanalysis. Br J Psychiatry. 2013;202(1):14-21.
Neuropsychiatric Disease and Treatment

\section{Publish your work in this journal}

Neuropsychiatric Disease and Treatment is an international, peerreviewed journal of clinical therapeutics and pharmacology focusing on concise rapid reporting of clinical or pre-clinical studies on a range of neuropsychiatric and neurological disorders. This journal is indexed on PubMed Central, the 'PsycINFO' database and CAS,
Dovepress

and is the official journal of The International Neuropsychiatric Association (INA). The manuscript management system is completely online and includes a very quick and fair peer-review system, which is all easy to use. Visit http://www.dovepress.com/testimonials.php to read real quotes from published authors. 